\title{
New Integral Inequalities for the Nevanlinna Characteristics of Meromorphic Functions
}

\author{
Md Mainul Islam ${ }^{1}$ \& A. N. M. Rezaul Karim² \\ ${ }^{1}$ Department of Computer Science and Engineering, Central Women's University, Bangladesh \\ ${ }^{2}$ Department of Computer Science and Engineering, International Islamic University Chittagong, Bangladesh \\ Correspondence: Md Mainul Islam, Department of Computer Science and Engineering, Central Women's \\ University, Bangladesh. E-mail: mainulmath83@yahoo.com
}

Received: October 29, 2014 Accepted: April 25, 2015 Online Published: July 13, 2015

doi:10.5539/ijsp.v4n3p99 URL: http://dx.doi.org/10.5539/ijsp.v4n3p99

\begin{abstract}
In this paper, we introduce a generalization of the Nevanlinna characteristics and give a short survey of classical and recent results on the representation of a meromorphic function in terms of such characteristics. And then we characterize the counting functions $N(r, f), N(r, a)$, and the characteristics functions $T(r, f), T(r, a)$ defined on a non-constant meromorphic $f(z)$. Besides this, we prove that the terms $N\left(e^{u}, f\right), N\left(e^{u}, a\right)$, $T\left(e^{u}, f\right)$, and $T\left(e^{u}, a\right)$ are convex functions for any real values of $u$. Finally, we derive some integral inequalities depending on these terms, analogous to well known Hadamard's inequality, by using elementary analysis.
\end{abstract}

Keywords: Analytic function, convex function, the Hermite-Hadamard integral inequality, meromorphic function, Nevanlinna characteristics, Carton's Identity.

\section{Introduction}

Convexity plays a central and fundamental role in mathematical finance, economics, engineering, management science, and optimization theory. In this paper, by meromorphic function we will always mean meromorphic function in the complex plane.

It is well-known that if the function $f: I \subseteq \mathbb{R} \rightarrow \mathbb{R}$ is convex then, for $a, b \in I$ with $a<b$, the following double inequality

$$
f\left(\frac{a+b}{2}\right) \leq \frac{1}{b-a} \int_{a}^{b} f(x) d x \leq \frac{f(a)+f(b)}{2} .
$$

holds. This is called the Hermite-Hadamard inequality. Since its innovation in 1893, Hadamard's inequality (Hadamard, 1893) has been proved to be one of the most practical inequalities in mathematical analysis. In recent years, a lot of refinements and generalizations of the Hermite-Hadamard inequality have been obtained for convex functions and its variant forms (Hadamard, 1893; Jiang, 2014; Jing, 2014; Lahiri, 1988; Mainul, 2013), and reference cited therein. Hadamard's inequalities deal with a convex function $f(x)$ on $[a, b] \in \mathbb{R}$ between the values of $f$ at the mid point $x=(a+b) / 2$ and the average of the values of $f$ at the endpoints $a$ and $b$ (Chen, 2012). The main principle of this paper is to establish some integral inequality involving the Nevanlinna characteristics of meromorphic functions. Throughout this note, we write $\mathbb{C}, \mathbb{R}, \mathbb{R}^{+}$for the set of complex numbers, the set of real numbers, and the set of non-negative real numbers, respectively.

\section{Preliminaries}

In this part some necessary definitions and theorems are mentioned which are closely connected to our main results discussed in (Titchmarsh, 1939; Polya, 1926; Hayman, 1964).

Definition 2.1 (Convex function) A convex function is a function whose value at the midpoint of every interval in its domain does not exceed the arithmetic mean of its values at the ends of the interval. More generally, a function $f(x)$ is convex on an interval $[a, b]$ if for any two points $x_{1}$ and $x_{2}$ in $[a, b]$ and any $\lambda$ where $0<\lambda<1$

$$
f\left(\lambda x_{1}+(1-\lambda) x_{2}\right) \leq \lambda f\left(x_{1}\right)+(1-\lambda) f\left(x_{2}\right) .
$$


Also, if $f(x)$ has a second derivative in $[a, b]$, then a necessary and sufficient condition for it to be convex on that interval is that the second derivative $f^{\prime \prime}(x) \geq 0$ for all $x$ in $[a, b]$.

Moreover, if the inequality above is strict for all $x_{1}$ and $x_{2}$, then $f(x)$ is called strictly convex. Besides that the function is called concave when the sign of the inequality is reversed.

Definition 2.2 (Meromorphic function) A complex function is said to be analytic on a region $R$, if it is complex differentiable at every point in $R$. The terms holomorphic function, differentiable function, and complex differentiable function are used interchangeably with "analytic function". If a complex function is analytic on a region $R$, it is infinitely differentiable in $R$. Also, a complex function may fail to be analytic at one or more points through the presence of singularities, or along lines or line segments through the presence of branch cuts.

A complex function that is analytic at all finite points of the complex plane is said to be entire. A single-valued function that is analytic in all but possibly a discrete subset of its domain, and at those singularities goes to infinity like a polynomial, is called a meromorphic function.

However, a meromorphic function therefore may only have finite-order, isolated poles and zeros and no essential singularities in its domain.

Example: all rational functions, $\tan z, \cot z, \frac{1}{e^{z}-1}$ etc.

Definition 2.3 (Transcendental meromorphic function) A meromorphic function other than a rational function must have an essential singularity at the point at infinity is called transcendental meromorphic functions.

Theorem 2.4 Every single-valued analytic function which is regular for every finite value of $\mathrm{z}$ and for $\mathrm{z}=\infty$, is a constant function.

Theorem 2.5 A function $\mathrm{f}(\mathrm{z})$ is regular everywhere except at infinity where it has a pole of order $\mathrm{n}$, is a polynomial of degree $n$.

Theorem 2.6 Every rational function is meromorphic in the extended plane. Also the converse, a function which is meromorphic in the extended plane is a rational function.

Theorem 2.7 (Expansion of meromorphic function) Let $f(z)$ be a non-constant meromorphic function, it's all singularities, except at infinity, are poles. For simplicity suppose that poles are simple. Let $a_{\mu}$ 's represent the poles and $b_{\mu}$ 's represent the residues, respectively. If $0 \leq\left|a_{1}\right| \leq\left|a_{2}\right| \leq \cdots$ then we introduce a sequence of closed contours $C_{n}$, such that $C_{n}$ inclued $a_{1}, a_{2}, \ldots, a_{n}$, but no other poles; and the minimum distance $R_{n}$ of $C_{n}$ from the origin tends to infinity with $n$, while $L_{n}$, the length of $C_{n}$, is $\mathrm{O}\left(R_{n}\right)$ i.e. $L_{n}=\mathrm{O}\left(R_{n}\right)$ and such that, on $C_{n} f(z)=\mathrm{O}\left(R_{n}\right)$, this implies that $f(z)$ is bounded on the system of closed contours $C_{n}$ taken as a whole under this conditions,

$$
f(z)=f(0)+\sum_{n=1}^{\infty} b_{n}\left(\frac{1}{z-a_{n}}+\frac{1}{a_{n}}\right)
$$

for all values of $z$, except the poles.

Theorem 2.8 (The Poisson-Jensen's formula) Let $f(z)$ be a non-constant meromorphic function. It has zeros at the points $a_{1}, a_{2}, \ldots, a_{m}$ and poles at the points $b_{1}, b_{2}, \ldots, b_{n}$ in the disc $|z| \leq R$, and this is analytic elsewhere inside disc $|z| \leq R$. Then

$$
\begin{gathered}
\ln \left|f\left(r e^{i \theta}\right)\right|=\frac{1}{2 \pi} \int_{0}^{2 \pi} \frac{\left(R^{2}-r^{2}\right) \ln \left|f\left(R e^{i \varphi}\right)\right|}{R^{2}-2 r R \cos (\theta-\varphi)+r^{2}} d \varphi \\
-\sum_{\mu=1}^{m} \ln \left|\frac{R^{2}-\bar{a}_{\mu} r e^{i \theta}}{R\left(r e^{i \theta}-a_{\mu}\right)}\right|+\sum_{\gamma=1}^{n} \ln \left|\frac{R^{2}-\bar{b}_{\gamma} r e^{i \theta}}{R\left(r e^{i \theta}-b_{\gamma}\right)}\right|
\end{gathered}
$$

for any $z=r e^{i \theta}(0 \leq r<R)$, except any poles and zeros of $f(z)$.

\section{The Nevanlinna Characteristics}

Let $f(z)$ be a meromorphic function. We shall introduce several real functions defined on $[0, \infty)$ which characterize the behavior of $f(z)$. The functions to be introduced will be called the Nevanlinna characteristics of $f(z)$.

Let $a$ be a complex number, and the real-valued function $n(r, f=a)$ defined by 


$$
n(r, f=a)=\left\{\begin{array}{lll}
n(r, f), & \text { for } & a=\infty, \\
n\left(r, \frac{1}{f-a}\right), & \text { for } & a \neq \infty .
\end{array}\right.
$$

and $n(0, f=a)=\left\{\begin{array}{lll}n(0, f), & \text { for } & a=\infty, \\ n\left(0, \frac{1}{f-a}\right), & \text { for } & a \neq \infty .\end{array}\right.$

This functions inform us how many poles and zeros of $f(z)$ are in the disc $|z| \leq r$. In this paper we use $n(r, a)$ instead of $n\left(r, \frac{1}{f-a}\right)$, here $n(r, f)$ and $n(r, a)$ represent the number of poles and zeros respectively. Similarly, Here $n(0, f)$ and $n(0, a)$ represent the number poles and zeros of $f(z)$ in the disc $|z| \leq \epsilon(\epsilon>$ $0)$, respectively. We assume that a pole of order $m$ contributes $m$ to the value of $n(r, f)$. It is clear that $n(r, f)$ and $n(r, a)$ are integer-valued, non-decreasing, and right semi-continuous on $[0, \infty)$. A point $r_{0} \epsilon(0, \infty)$ is a point of discontinuity for $n(r, f)$ if and only if the disk $|z|=r_{0}$ contains poles of $f(z)$, the value of the jump at $r_{0}$ is equal to the number of such poles. The set of points of discontinuity of $n(r, f)$ cannot have limit points in $[0, \infty)$, therefore on each interval $\left[x_{1}, x_{2}\right] \subset[0, \infty)$ the function $n(r, f)$ is piecewise constant. However similar properties hold for $n(r, a)$. It is clear that the functions $n(r, f)$ and $n(r, a)$ are non-decreasing continuous functions for any positive real values of $r$.

Let $a$ be a complex number and let the counting function $N(r, f=a)$ be defined by

$$
= \begin{cases}N(r, f)=\int_{0}^{r} \frac{n(t, a)-n(0, a)}{t} d t+n(0, a) \ln r, & \text { for } a=\infty, \\ N\left(r, \frac{1}{f-a}\right)=\int_{0}^{r} \frac{n(t, a)-n(0, a)}{t} d t+n(0, a) \ln r, & \text { for } a \neq \infty .\end{cases}
$$

This characteristics also describes the location of poles and zeros of $f(z)$. The counting functions $N(r, f)$, and $N(r, f)$ are real-valued, non-decreasing continuous functions on $(0, \infty)$.

Let $f(z)$ be a non-constant meromorphic function [11]. Assume $a_{\mu}$, and $b_{\mu}$ represent the zeros and the poles of $f(z)$ in the disc $|z| \leq r$, respectively. If $0 \leq\left|a_{1}\right| \leq\left|a_{2}\right| \leq \cdots$ and If $0 \leq\left|b_{1}\right| \leq\left|b_{2}\right| \leq \cdots$, then we obtain that

$$
\begin{gathered}
\sum_{\mu=1}^{n} \ln \frac{R}{\left|a_{\mu}\right|}=\sum_{\mu=1}^{n} \ln \frac{R}{r_{\mu}}=\ln \frac{R^{n}}{r_{1} r_{2} \ldots r_{n}}, \\
=n \ln R-\sum_{m=1}^{n} \ln r_{m}, \\
=n \ln R-n \ln r_{n}+n \ln r_{n}-\sum_{m=1}^{n} \ln r_{m}, \\
=n\left(\ln R-\ln r_{n}\right)+\sum_{m=1}^{n-1} m\left(\ln r_{m+1}-\ln r_{m}\right), \\
=n \int_{r_{n}}^{R} \frac{1}{x} d x+\sum_{m}^{n-1} m \int_{r_{m}}^{r_{m+1}} \frac{1}{x} d x, \\
=\int_{r_{n}}^{R} \frac{n(x, a)}{x} d x+\sum_{m}^{n-1} \int_{r_{m}}^{r_{m+1}} \frac{n(x, a)}{x} d x,
\end{gathered}
$$




$$
\begin{aligned}
=\int_{r_{1}}^{R} \frac{n(x, a)}{x} d x, \\
=\int_{\epsilon}^{R} \frac{n(x, a)}{x} d x, \quad\left[\text { since } n(x, a)=0, \text { for any } x \in\left(0, r_{1}\right)\right] \\
=\int_{0}^{R} \frac{n(x, a)-n(0, a)}{x} d x, \\
=N(R, a)-n(0, a) \ln r .
\end{aligned}
$$

Similarly, for the poles we get,

$$
\sum_{\gamma=1}^{n} \ln \frac{R}{\left|b_{\gamma}\right|}=N(R, f)-n(0, a) \ln r .
$$

Now, if we define

$$
\ln ^{+} x=\left\{\begin{array}{l}
\ln x, \quad x \geq 1 \\
0, \quad 0 \leq x<1
\end{array}\right.
$$

Then the following properties are obvious:
a. $\ln ^{+} x \geq 0$
if $x \geq 0$,
b. $\ln ^{+} x \geq \ln x$
if $x>0$,
c. $\ln ^{+} x \geq \ln ^{+} y$
if $x>y$,
d. $\quad \ln x=\ln ^{+} x-\operatorname{lm}^{+} \frac{1}{x}$
if $x>0$.

Let $a$ be a complex number and let the proximity function $m(r, f=a)$ be defined by (Jing, 2014).

$$
\begin{gathered}
m(r, f=a) \\
= \begin{cases}m(r, f)=\frac{1}{2 \pi} \int_{0}^{2 \pi} \ln ^{+}\left|f\left(R e^{i \varphi}\right)\right| d \varphi, & \text { for } a=\infty, \\
m\left(r, \frac{1}{f-a}\right)=\frac{1}{2 \pi} \int_{0}^{2 \pi} \ln ^{+} \frac{1}{\left|f\left(R e^{i \varphi}\right)-a\right|} d \varphi, & \text { for } a \neq \infty .\end{cases}
\end{gathered}
$$

This function characterizes the growth of the function $f(z)$. Also, it is a continuous function for any positive real values of $r$. In this paper we use $m(r, a)$ instead of $\left(r, \frac{1}{f-a}\right)$.

Characteristics of $N(r, f)$ is useful because it is related, in a natural way, to the Jensen formula. The Jensen formula, for $f(0) \neq 0$ or $\infty$, can be written as

$$
m(R, f)+N(R, f)=m(R, a)+N(R, a)+\ln |f(0)| .
$$

Also. for $f(0)=0$ or $\infty$, i.e. if $f(z)$ has a zero of order $\lambda$ or a pole of order $\lambda$ at $z=0$. Then

when $\quad c_{\lambda}$ is coefficient of $z^{\lambda}$ in the expansion of $f(z)$.

$$
m(R, f)+N(R, f)=m(R, a)+N(R, a)+\ln \left|c_{\lambda}\right|,
$$

Let $a$ be a complex number, then the Nevanlinna characteristics function $T(r, f=a)$ on the meromorphic function $f(z)$ are define by

$$
T(r, f=a)= \begin{cases}T(r, f)=m(r, f)+N(r, f) & \text { for } \quad a=\infty \\ T(r, a)=m(r, a)+N(r, a) & \text { for } \quad a \neq \infty\end{cases}
$$

This is clear that $T(R, f)$, and $T(R, a)$ are real-valued continuous of $r \in[0, \infty)$. (Hayman, 1926)

\section{Auxiliary Lemmas and Theorems}

Theorem 4.1 (The Nevanlinna's first fundamental theorem) Let $\mathrm{f}(\mathrm{z})$ be a non-constant meromorphic function, and $a$ is a complex number. Then, for $f(0) \neq a, \infty$, we have 


$$
T(R, a)=T(R, f)-\ln |f(0)-a|+\epsilon(a, R) .
$$

where, $|\epsilon(\mathrm{a}, \mathrm{R})| \leq \ln ^{+}|\mathrm{a}|+\ln 2, \quad[\epsilon(\mathrm{a}, \mathrm{R})$, for each $\mathrm{a}$, is bounded as $\mathrm{R}$ goes to infinity]. (Hayman, 1926)

Theorem 4.2 (Cartan's identity) Let $f(z)$ be a meromorphic function defined on the disc $|z| \leq R$. Then, for $f(0) \neq 0, \infty$, we obtain

$$
\mathrm{T}(\mathrm{r}, \mathrm{f})=\frac{1}{2 \pi} \int_{0}^{2 \pi} \mathrm{N}\left(\mathrm{r}, \mathrm{e}^{\mathrm{i} \theta}\right) \mathrm{d} \theta+\ln ^{+}|\mathrm{f}(0)|, \quad(0<r<R) .
$$

The expression of $T(r, f)$ by an integral is known as Cartan's identity (due to H.Cartan). (Hayman, 1926)

Lemma 4.3 The upper bound of $m(r, f)$ can be given by the relation (Hayman, 1926).

$$
\frac{1}{2 \pi} \int_{0}^{2 \pi} m\left(r, e^{i \theta}\right) d \theta \leq \ln 2 .
$$

Lemma 4.4 (The relationships between the maximum modulus and the characteristic function of a regular function) If $f(z)$ is an analytic function defined on the disc $|z| \leq R,(0<R \leq+\infty)$, and the maximum modulus defined by

$$
M(r, f)=\max _{|z|=r}|f(z)|
$$

Then the following inequality holds

$$
T(r, f) \leq \ln ^{+} M(r, f) \leq \frac{R+r}{R-r} T(r, f), \quad(0<r<R) .
$$

Lemma 4.5 Let $f(z)$ be a non-constant meromorphic function, and $a$ is a complex number. Let the counting functions $N(r, f), N(r, a)$, and the characteristics functions $T(r, f), T(r, a)$ be defined on $f(z)$. Then these four functions are convex functions of $\ln r$, for $0<r<\infty$.

Proof: Using the definition (1.1) it is sufficient to show that a real function $\phi(x)$ is convex if and only if $\phi^{\prime \prime}(x) \geq 0$, that is $\phi^{\prime}(x)$ is non-decreasing.

We have

$$
N(r, f)=\int_{0}^{r} \frac{n(t, f)-n(0, f)}{t} d t+n(0, f) \ln r
$$

Differentiate with respect to $\ln r$, then we get

$$
\begin{aligned}
\frac{d N(r, f)}{d \ln r}= & \frac{d}{d \ln r}\left[\int_{0}^{r} \frac{n(t, f)-n(0, f)}{t} d t+n(0, f) \ln r\right] \\
= & \frac{d}{d \ln r} \int_{0}^{r} \frac{n(t, f)-n(0, f)}{t} d t+n(0, f) \\
= & \frac{d}{d \ln r} \int_{0}^{r}\{n(t, f)-n(0, f)\} d \ln t+n(0, f) \\
= & n(r, f)-n(0, f)+n(0, f)=n(r, f) .
\end{aligned}
$$

Therefore, $N(r, f)$ is a convex function of $\ln r$, Similarly, we show that $N(r, a)$ is a convex function of $\ln r$. Furthermore, for $-\infty<u<\infty$, we obtain

$$
N\left(e^{u}, f\right)=\int_{-\infty}^{u}\left[n\left(e^{t}, f\right)-n(0, f)\right] d t+n(0, f) u .
$$

Then $N\left(e^{u}, f\right)$ is an integral of a non-decreasing real-valued continuous function of $u$, and it is convex function of $\mathrm{u}$. Therefore, $N(r, f)$, and $N(r, a)$ are convex functions of $\ln r, r \in(0, \infty)$.

By the Cartan's identity relation, we have

$$
T(r, f)=\frac{1}{2 \pi} \int_{0}^{2 \pi} N\left(r, e^{i \theta}\right) d \theta+\ln ^{+}|f(0)|
$$

Differentiate with respect to $\ln r$, then we get 


$$
\begin{aligned}
\frac{d T(r, f)}{d \ln r}= & \frac{d}{d \ln r}\left[\frac{1}{2 \pi} \int_{0}^{2 \pi} N\left(r, e^{i \theta}\right) d \theta+\ln ^{+}|f(0)|\right] \\
= & \frac{d}{d \ln r}\left[\frac{1}{2 \pi} \int_{0}^{2 \pi} N\left(r, e^{i \theta}\right) d \theta\right]+0 \\
& =\frac{1}{2 \pi} \int_{0}^{2 \pi} \frac{d}{d \ln r} N\left(r, e^{i \theta}\right) d \theta \\
& =\frac{1}{2 \pi} \int_{0}^{2 \pi} n\left(r, e^{i \theta}\right) d \theta
\end{aligned}
$$

The value of right-hand side integral is always positive, because $n(r, f)>0$, and is a non-decreasing real-valued continuous function of $r$. Therefore, we conclude that $T(r, f)$ is a convex functions of $\ln r$. Moreover, we also show that $T\left(r^{u}, f\right),-\infty<u<\infty$, is a convex function of $u$. Similarly, we show that $T(r, a)$ and $T\left(e^{u}, a\right)$ are two convex function of $\ln r$ and $u$, respectively.

\section{Main Results}

Theorem 5.1 Let $f(z)$ be a non-constant meromorphic function, and $a$ be a complex number. Let the counting functions $N(r, f)$, and $N(r, a)$ be defined on $f(z)$. Then, for any $x_{1}, x_{2} \in I \subset[0, \infty)$ with $x_{1}<x_{2}$, we get the following inequalities

$$
N\left(\frac{x_{2}+x_{1}}{2}, f\right) \leq \frac{1}{x_{2}-x_{1}} \int_{x_{1}}^{x_{2}} N\left(e^{t}, f\right) d t \leq \frac{N\left(x_{2}, f\right)+N\left(x_{1}, f\right)}{2}
$$

and, $\quad N\left(\frac{x_{2}+x_{1}}{2}, a\right) \leq \frac{1}{x_{2}-x_{1}} \int_{x_{1}}^{x_{2}} N\left(e^{t}, a\right) d t \leq \frac{N\left(x_{2}, a\right)+N\left(x_{1}, a\right)}{2}$.

Proof: Consider the real function $\quad N: I \subset \mathbb{R} \rightarrow \mathbb{R}$, defined by

$$
= \begin{cases}N\left(e^{u}, f=a\right) & \text { for } \mathrm{a}=\infty, \\ N\left(e^{u}, a\right)=\int_{-\infty}^{u}\left[n\left(e^{t}, a\right)-n(0, a)\right] d t+n(0, a) u & \text { for } a \neq \infty .\end{cases}
$$

Using lemma (4.5), we say that $N\left(e^{u}, f\right)$ and $N\left(e^{u}, a\right)$ are two convex functions of $u$, for $-\infty<u<\infty$. Now using the Hermite-Hadamard inequality on convex functions, for any $x_{1}, x_{2} \in I \subset[0, \infty)$ with $x_{1}<x_{2}$, we derive the following double inequalities

$$
N\left(\frac{x_{2}+x_{1}}{2}, f\right) \leq \frac{1}{x_{2}-x_{1}} \int_{x_{1}}^{x_{2}} N\left(e^{t}, f\right) d t \leq \frac{N\left(x_{2}, f\right)+N\left(x_{1}, f\right)}{2},
$$

and, $\quad N\left(\frac{x_{2}+x_{1}}{2}, a\right) \leq \frac{1}{x_{2}-x_{1}} \int_{x_{1}}^{x_{2}} N\left(e^{t}, a\right) d t \leq \frac{N\left(x_{2}, a\right)+N\left(x_{1}, a\right)}{2}$.

This completes the proof of theorem (5.1).

Theorem 5.2 Let $f(z)$ be a non-constant meromorphic function, and $a$ be a complex number. Let the counting functions $T(r, f)$, and $T(r, a)$ be defined on $f(z)$. Then, for any $x_{1}, x_{2} \in I \subset[0, \infty)$ with $x_{1}<x_{2}$, we get the following inequalities

$$
T\left(\frac{x_{2}+x_{1}}{2}, f\right) \leq \frac{1}{x_{2}-x_{1}} \int_{x_{1}}^{x_{2}} T\left(e^{u}, f\right) d u \leq \frac{T\left(x_{2}, f\right)+T\left(x_{1}, f\right)}{2},
$$

and, $T\left(\frac{x_{2}+x_{1}}{2}, a\right) \leq \frac{1}{x_{2}-x_{1}} \int_{x_{1}}^{x_{2}} T\left(e^{u}, a\right) d u \leq \frac{T\left(x_{2}, a\right)+T\left(x_{1}, a\right)}{2}$.

Proof: Consider the real function $\quad T: I \subset \mathbb{R} \rightarrow \mathbb{R}$, defined by

$$
T\left(e^{u}, f=a\right)= \begin{cases}T\left(e^{u}, f\right)=m\left(e^{u}, f\right)+N\left(e^{u}, f\right), & \text { for } \mathrm{a}=\infty, \\ T\left(e^{u}, a\right)=m\left(e^{u}, a\right)+N\left(e^{u}, a\right), & \text { for } a \neq \infty .\end{cases}
$$


Using lemma (4.5), we say that the Nevanlinna Characteristics functions $T\left(e^{u}, f\right)$, and $T\left(e^{u}, a\right)$ are two convex functions of $u$, for $-\infty<u<\infty$. Now using the Hermite-Hadamard inequality on convex functions, for any $x_{1}, x_{2} \in I \subset[0, \infty)$ with $x_{1}<x_{2}$, we derive the following double inequalities

$$
T\left(\frac{x_{2}+x_{1}}{2}, f\right) \leq \frac{1}{x_{2}-x_{1}} \int_{x_{1}}^{x_{2}} T\left(e^{t}, f\right) d t \leq \frac{T\left(x_{2}, f\right)+T\left(x_{1}, f\right)}{2},
$$

and, $\quad T\left(\frac{x_{2}+x_{1}}{2}, a\right) \leq \frac{1}{x_{2}-x_{1}} \int_{x_{1}}^{x_{2}} T\left(e^{t}, a\right) d t \leq \frac{T\left(x_{2}, a\right)+T\left(x_{1}, a\right)}{2}$.

This completes the proof of theorem (5.2).

\section{References}

Chen, Y. (2012). Multi-dimensional Hadamard's inequalities. TJM, 43(1), 1-10, Spring.

Copson, E. T. (1935). An introduction to the theory of functions of a complex variable, Oxford University Press.

Dragomir, S. S. (1990). Two refinements of Hadamard's inequalities. Coll. Of Sci. Pap. Of the Fac.of Sci., Kragujevac (Yougoslavia), 11, 23-26.

Dragomir, S. S. (1992). On Hadamard's inequalities for convex functions. Math. Balkanica, 6(4), 251-222.

Hayman, W. K. (1964). Meromorphic functions. Oxford.

Indrajit, L. (1988). Generalized order of the derivative of a meromorphic function. SJM, 14(1), 85-92.

Islam, M. M. (2014). A Generalized Extension of the Hadamard-type Inequality for a Convex Function Defined on the Minimum Modulus of Integral Functions. Maxwell Scientific Organization. Res. J. Appl. Sci. Eng. Technol., 8(5), 595-599.

Islam, M. M., \& Rezaul Karim, A. N. M. (2013). An extension of the Hadamard-Type Inequality for a convex function defined on Modulus of complex integral functions. JMR, 5(3). http://dx.doi.org/10.5539/jmr.v5n3p92

Jiang, W. D., Niu, D. W., \& Qi, F. (2014). Some inequalities of Hermite-Hadamard type for $r-\varphi$ connvex functions. TJM, 45(1), 31-38.

Kirmaci, U. S., \& Dikici, R. (2013). On some Hermite-Hadamard type inequalities for twice differentiable mappings and applications. TJM, 44(1), 41-51.

Li, J., Zhang, J., \& Liao, L. (2014). On growth of meromorphic solutions of complex function difference equations, Abstract and Applied Analysis, Hindawi Publishing Corporation. http://dx.doi.org/10.1155/2014/828746.

Li, X. M., Yi, H. X., \& Li, W. L. (2014). Value distribution of certain difference polynomials of meromorphic functions. RMJM, 44(2).

MevlütTunc. (2012). On some new inequalities for convex functions. Turk J Math, 36, 245-251.

Polya, G. (1926). On the minimum modulus of integral functions. Journal London Math. Soc, 1, 78-86.

Qi, X., \& Dou, J. (2013). Zeros and Uniqueness of difference polynomials of meromorphic functions. Kyungpook Math. J., 53, 541-552.

Titchmarsh, E. C. (1932). The theory of function, Oxford University Press.

Yang, G. S., \& Hong, M. C. (1997). A note on Hadamard's inequality, Tamkang. J. Math., 28, 33-77.

\section{Copyrights}

Copyright for this article is retained by the author(s), with first publication rights granted to the journal.

This is an open-access article distributed under the terms and conditions of the Creative Commons Attribution license (http://creativecommons.org/licenses/by/3.0/). 